\title{
The prognostic impact of CD49d protein and mRNA expression in patients with chronic lymphocytic leukaemia
}

Maciej Putowski ${ }^{1}$, Katarzyna Skórka' ${ }^{1}$, Monika Włodarczyk ${ }^{1}$, Waldemar Tomczak² ${ }^{2}$ Edyta Subocz ${ }^{3,4}$, Janusz Hałka ${ }^{3,4}$, Krzysztof Giannopoulos ${ }^{1,5}$

\author{
${ }^{1}$ Department of Experimental Hematooncology, Medical University of Lublin, Poland \\ ${ }^{2}$ Chair and Clinic of Hematooncology and Bone Marrow Transplantation, Medical \\ University of Lublin, Poland, Poland \\ ${ }^{3}$ Department of Internal Medicine and Hematology, Military Institute of Medicine, \\ Poland \\ ${ }^{4}$ Department of Hematology, Warmian-Masurian Cancer Centre of The Ministry of The \\ Interior and Administration's Hospital, Poland \\ ${ }^{5}$ Department of Hematology, St. John's Cancer Centre, Poland
}

Submitted: 9 November 2020

Accepted: 19 November 2020

Arch Med Sci 2020;

DOI: https://doi.org/10.5114/aoms/130646

Copyright $\odot$ Termedia \& Banach

\section{Abstract}

Introduction: This research aimed to study CD49d expression - a potential prognosis marker in chronic lymphocytic leukaemia (CLL) patients - at the protein and mRNA levels. It was analysed in terms of time to first treatment and compared to currently known prognostic markers and novel molecular markers.

Material and methods: Using samples from 199 newly diagnosed CLL patients, we conducted immunophenotypical analyses of CD49d with flow cytometry, and assessed its expression on the mRNA level using quantitative polymerase chain reaction (PCR).

Results: Cytometric analysis showed significantly higher expression of CD49d protein in ZAP-70+ (cut-off of $20 \%$ ) patients than that in ZAP-70 patients (18.52 vs. 6.57, $p=0.028)$, and a tendency of higher CD49d expression in CD38+ patients than in CD38 patients (20.48 vs. $7.25, p=0.072$ ). CD49d expression significantly correlated with serum $\beta 2$-microglobulin $(r=0.273, p=0.012)$ and lactate dehydrogenase activity $(r=0.159$, $p<0.01)$. Analysed in subgroups divided according to novel mutations, CD49d expression showed a tendency to be higher in MUT SF3B1 (93.98 vs. $7.86, p=0.06)$ and MUT NOTCH1 (18.52 vs. 7.86, $p=0.140$ ) groups than in the corresponding UNMUT groups. CD49d expression did not affect the time to first treatment at protein or mRNA level.

Conclusions: High CD49d expression at the protein level accompanies aggressive biological markers in CLL patients, indicating its prognostic potential. It can thus be used to identify patients with poor clinical CLL prognosis.

Key words: chronic lymphocytic leukaemia, NOTCH1, CD49d, SF3B1.

\section{Introduction}

Chronic lymphocytic leukaemia (CLL) is a heterogeneous disease characterised by the clonal expansion of mature, typically CD5-positive B cells that accumulate in the blood, bone marrow, and lymphoid organs.

\author{
Corresponding author: \\ Prof. Krzysztof Giannopoulos \\ Department of Experimental \\ Hematooncology, \\ Medical University of Lublin \\ Chodzki 1, 20-093 Lublin \\ Poland \\ Phone: 48814486632 \\ Fax: + 48814486634 \\ E-mail: krzysztof. \\ giannopoulos@gmail.com
}


CLL cells are defined by a distinctive immunophenotype of the surface antigen CD5 together with the B-cell antigens CD19, CD20, and CD23 [1].

The biological heterogeneity of the disease including chromosomal alterations, gene mutations, the immunoglobulin heavy-chain variable region (IGHV) mutation status, as well as immunophenotypic features - determines its variable clinical course, with patient survival ranging from months to decades [1, 2]. The currently most relevant prognostic score is the CLL International Prognostic Index (CLL-IPI), which classifies patients based on clinical stage, age, IGHV mutational status, serum $\beta 2$-microglobulin, and TP53 dysfunction, summing their hierarchical values based on the hazard ratios for OS [3]. During CLL diagnosis, other prognostic markers are used to predict the outcomes of the patients, the expression of zeta-chain-associated protein kinase 70 (ZAP-70), the expression of $\mathrm{CD} 38$, the concentration of lactate dehydrogenase level (LDL), and chromosome aberrations [4]. Hepatitis B surface antigen positivity is another unfavourable prognostic factor that is insufficiently screened in the Polish population of CLL patients [5].

Despite the wide range of markers in CLL, particularly at an early stage, currently available prognostic factors often show low efficiency in predicting the course of the disease. Therefore, more efficient biomarkers are still being sought [6]. To date, there is no comprehensive hierarchical model incorporating all markers.

Recent studies have pointed to the surface adhesion molecule CD49d as one of the strongest of the poor prognostic factors in CLL $[7,8]$. CD49d - the $\alpha$ chain of the $\alpha_{4} \beta_{1}$ integrin heterodimer, also known as very late antigen 4 (VLA-4), coded by the ITGA4 gene - is involved in forming supportive microenvironmental interactions of CLL cells and accessory cells such as nurse-like cells, vascular endothelial cells, dendritic cells, and T cells $[9,10]$. Functionally, by binding to fibronectin and vascular-cell adhesion molecule-1 (VCAM-1), CD49d mediates cell-cell and cell-matrix interactions. CD49d plays crucial roles in leucocyte trafficking, activation, and survival. It also mediates interactions between leucocytes and stromal cells of marrow or the germinal centre of lymphoid follicles [10-14]. Additionally, CD49d promotes B-cell survival via upregulation of $\mathrm{BCl}-2$ family members, which may determine progression of CLL [14]. CLL cells have also been found to require CD49d engagement to undergo transendothelial migration and enter the proliferation centres of lymph nodes $[15,16]$. Strati et al. [17] showed that CD49d expression was associated with nodal presentation and the subsequent development of lymphadenopathy in CLL patients. Furthermore, the effec- tiveness of blocking CD49d has been also studied in multiple sclerosis and acute leukaemia with the use of natalizumab - monoclonal antibody against CD49d and ATL1102 - second-generation antisense oligonucleotide to CD49d RNA [18].

In order to be effective, a new negative prognostic biomarker in CLL should also correlate with well-established markers for unfavourable prognosis. According to recent research, this is the case with CD49d, which has shown links with high ZAP70 and CD38 expression, unmutated IGHV genes, trisomy 12 , high $\beta-2$ microglobulin, and LDL $[7,19]$. Bulian et al. [8] reported that CD49d showed the predictive value of overall survival and treatment-free survival in CLL patients; they also classified CD49d as the strongest flow cytometry prognostic marker. Another study revealed the association of CD49d with the presence of NOTCH1 (neurogenic locus notch homologue protein 1) mutations [20]. These studies suggest that CD49d has potential predictive value in CLL, but due to the insufficient number of studies validating features of CD49d and its potential utility in the management of CLL further research is required.

This study aims to contribute to this knowledge by studying the expressions of CD49d protein and mRNA in a cohort of newly diagnosed Polish CLL patients. To distinguish between CD49d-positive and -negative patients when testing the prognostic impact of CD49d protein, most previous studies have recommended the cut-off of $30 \%$ of positive cells $[7,8,10,19]$. As benchmarks, we used the most common currently available prognostic factors in CLL: ZAP-70, CD38, IGHV gene mutational status, and IGHV subsets; and new mutations, such as NOTCH1, SF3B1 (splicing factor 3B subunit 1), and MYD88 (myeloid differentiation primary response gene 88 ). We also assessed the prognostic significance of CD49d in terms of the time to first treatment (TTFT).

\section{Material and methods}

\section{Participants}

The study used 199 newly diagnosed and previously untreated CLL patients. The cohort included 79 women and 120 men, with a median age of 66 (range 38-90) years. The prognostic significance in terms of TTFT was clinically assessed for 90 patients. The time to first treatment was defined as the time interval between the date of diagnosis and the date of first CLL treatment administered according to the International Workshop on Chronic Lymphocytic Leukaemia (iwCLL) treatment indications criteria [1]. The patients' detailed clinical characteristics are displayed in Table I. Ethical approval was granted by local review committees, and written informed consent 
was collected from each participant according to the principles of the Declaration of Helsinki.

\section{Peripheral blood mononuclear cell isolation}

From the 199 patients, we collected peripheral blood samples. Peripheral blood mononuclear cells were isolated using the intensity gradient centrifuge method, using Ficoll (Biochrom, Germany). Next, the cells were washed with PBS (Biochrom, Germany). The viability of peripheral blood mononuclear cells (PBMCs) was more than 95\%. Peripheral blood mononuclear cells were cryopreserved at $-80^{\circ} \mathrm{C}$ until the time of analysis.

\section{mRNA extraction and reverse transcription}

Total RNA isolation from PBMCs was performed using QIAamp RNA Kit (Qiagen, Netherlands), according to the manufacturer's protocol. Concentrations and purities were measured using BioSpec-nano (Thermo Scientific, USA). From each sample, $1 \mu \mathrm{g}$ of total RNA was reverse transcribed to $20 \mu \mathrm{l}$ of cDNA using a QuantiTect Reverse Transcription Kit (Qiagen, Netherlands). Quantitative reverse transcriptase-polymerase chain reaction (qRT-PCR) reactions used $1 \mu$ of cDNA per sample.

\section{Quantitative reverse transcriptase- polymerase chain reaction-}

The quantity of the mRNA expression of the transcript of CD49d (ITGA4) was assessed in each of the 199 CLL patients using qRT-PCR with the TaqMan Gene Expression Assays methodology (Applied Biosystems, USA). The procedure was performed according to the manufacturer's protocols. The end volume of a particular PCR reaction per well was $20 \mu \mathrm{l}$, which included $10 \mu \mathrm{l}$ of TaqMan Universal PCR Mastermix (Applied Biosystems, USA), $1 \mu \mathrm{l}$ of Taqman assay for ITGA4 with the specific primers and probe (Thermofisher scientific, USA), $8 \mu$ l RNase-Free water (Qiagen, Netherlands), and $1 \mu \mathrm{l}$ of cDNA. Distilled water was used as a negative control.

As a constitutively expressed housekeeping gene, glyceraldehyde-3-phosphate dehydrogenase $(G A P D H)$ was used. Expression of GAPDH was assessed using Taqman probes with TaqMan Universal PCR Master Mix (Applied Biosystems, USA) as well as TaqMan GAPDH Control Reagents Human Assays (Applied Biosystems, USA). The procedure was performed according to the manufacturer's protocols. The end volume of a particular PCR reaction per well was $25 \mu \mathrm{l}$, which included $12.5 \mu \mathrm{l}$ of TaqMan Universal PCR Mastermix (Applied Biosystems, USA), $0.5 \mu \mathrm{l}$ of forward primer as well as 0.5 $\mu \mathrm{l}$ of reverse primer for GAPDH (Applied Biosystems, USA), $10 \mu$ l RNase-Free water (Qiagen, Netherlands), $0.5 \mu \mathrm{l}$ JOE probe, and $1 \mu \mathrm{l}$ of cDNA.
Table I. Clinical characteristic of patients

\begin{tabular}{|c|c|c|}
\hline & $\begin{array}{l}\text { CD49d } \\
\text { mRNA }\end{array}$ & $\begin{array}{l}\text { CD49d } \\
\text { protein }\end{array}$ \\
\hline Number of patients & 199 & 106 \\
\hline \multicolumn{3}{|l|}{ Sex } \\
\hline female & 79 & 38 \\
\hline male & 120 & 68 \\
\hline Median age (years) & 66 & 66 \\
\hline Age range (years) & $38-90$ & $38-90$ \\
\hline \multicolumn{3}{|l|}{ CD49d mRNA } \\
\hline high & 99 & 47 \\
\hline low & 100 & 51 \\
\hline not available & $\mathrm{n} / \mathrm{a}$ & 8 \\
\hline \multicolumn{3}{|c|}{ CD49d protein (cut-off 30\%) } \\
\hline positive ( $\geq 30 \%$ ) & 36 & 36 \\
\hline negative $(<30 \%)$ & 70 & 70 \\
\hline not available & 93 & $\mathrm{n} / \mathrm{a}$ \\
\hline \multicolumn{3}{|l|}{ ZAP-70 (cut-off 20\%) } \\
\hline positive ( $\geq 20 \%$ ) & 58 & 31 \\
\hline negative $(<20 \%)$ & 104 & 56 \\
\hline not available & 37 & 19 \\
\hline \multicolumn{3}{|l|}{ CD38 (cut-off 30\%) } \\
\hline positive ( $\geq 30 \%$ ) & 52 & 27 \\
\hline negative $(<30 \%)$ & 116 & 59 \\
\hline not available & 31 & 20 \\
\hline \multicolumn{3}{|l|}{ IGHV mutation status } \\
\hline mutated & 94 & 49 \\
\hline unmutated & 102 & 54 \\
\hline not available & 3 & 3 \\
\hline
\end{tabular}

ZAP-70 - zeta-chain-associated protein kinase 70, IGHV immunoglobulin heavy-chain variable region gene, n/a - not applicable.

Thermocycling program was set for 40 cycles of $15 \mathrm{sec}$ at $95^{\circ} \mathrm{C}, 1 \mathrm{~min}$ at $60^{\circ} \mathrm{C}$, with an initial denaturation step at $95^{\circ} \mathrm{C}$ for 10 min using the 7300 Real Time PCR System (Applied Biosystems, Life Technologies Corporation, USA).

The particular gene expression levels of ITGA4 were calculated as an inverse ratio of the difference in cycle threshold $(\Delta \mathrm{Ct})$, where $\Delta \mathrm{Ct}$ is the $\mathrm{Ct}$ value of the transcript of CD49d (ITGA4) expression minus the Ct value of GAPDH.

\section{Immunophenotypic analyses of CD49d,} ZAP-70, and CD38-

Flow cytometry was used to characterise the surface expression of Cd49d on CLL cells using combination of fluorescent-labelled monoclonal antibodies: anti-CD19 FITC (BD Biosciences, USA), 
anti-CD5 PerCP5 (BD Biosciences, USA), and anti-CD49d APC (BD Biosciences, USA). A fluorescence minus one (FMO) control was used for PE. Samples were measured using an FACSCLyric (BD Biosciences, USA) flow cytometer. Data were analysed using the BD FACSuite software (BD Biosciences, USA). A minimum of 100,000 cells were collected and analysed. The representative flow cytometry analysis is presented in Figure 1.

Evaluation of CD38 and ZAP-70 expression in CD19+/CD5+ leukemic cells in all tested samples was performed according to the previously described procedure [21]. Based on a CD38 cut-off value of $30 \%$, CLL patients were categorised into CD38-positive ( $\geq 30 \%)$ and CD38-negative subgroups (<30\%). For ZAP-70 the cut-off value was defined as $20 \%$, and patients were categorised into ZAP-70-positive ( $\geq 20 \%$ ) and ZAP-70-negative $(<20 \%)$ subgroups. The cut-off values used in the present report were selected in accordance with previously published studies [21].

\section{NOTCH1, MYD88, SF3B1, and IGHV mutations}

NOTCH1 c.7544 7545delCT in PEST domain (exon 34) and MYD88 L265P mutations were in vestigated by amplification refractory mutation system PCR. SF3B1 mutations (K700, E622/R625, and $H 662 / K 666)$ were confirmed by Sanger sequencing method. Detection of SF3B1, NOTCH1, MYD88 L265P, and IGHV mutation status were assessed as previously described in detail [22] A cut-off of $98 \%$ germline homology was used to assess IGHV mutation status. The sequences with a germline homology of $98 \%$ or higher were considered unmutated, and those with a homology less than $98 \%$ were considered mutated.

\section{Statistical analysis}

Statistical analyses were performed using GraphPad Prism 8 (La Jolla, CA, United States). Because the data did not follow the normal distribution, we used nonparametric methods. Dif-

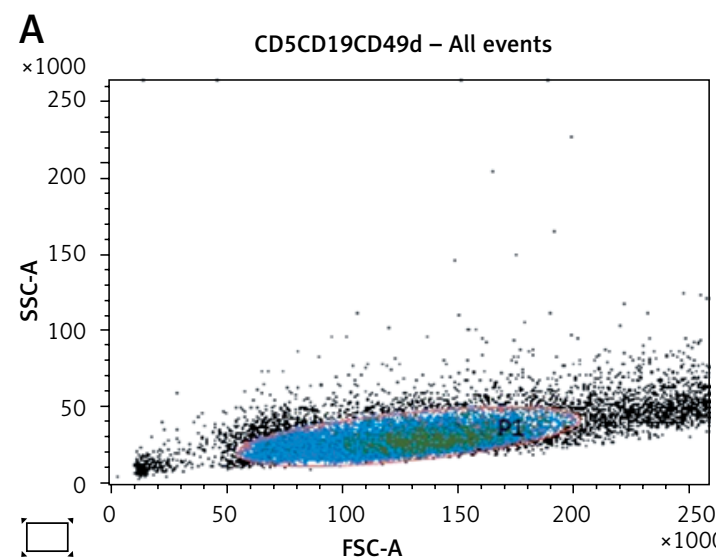

B

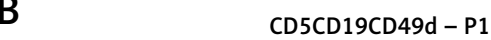

C FMO dla PE - CD19+CD5+
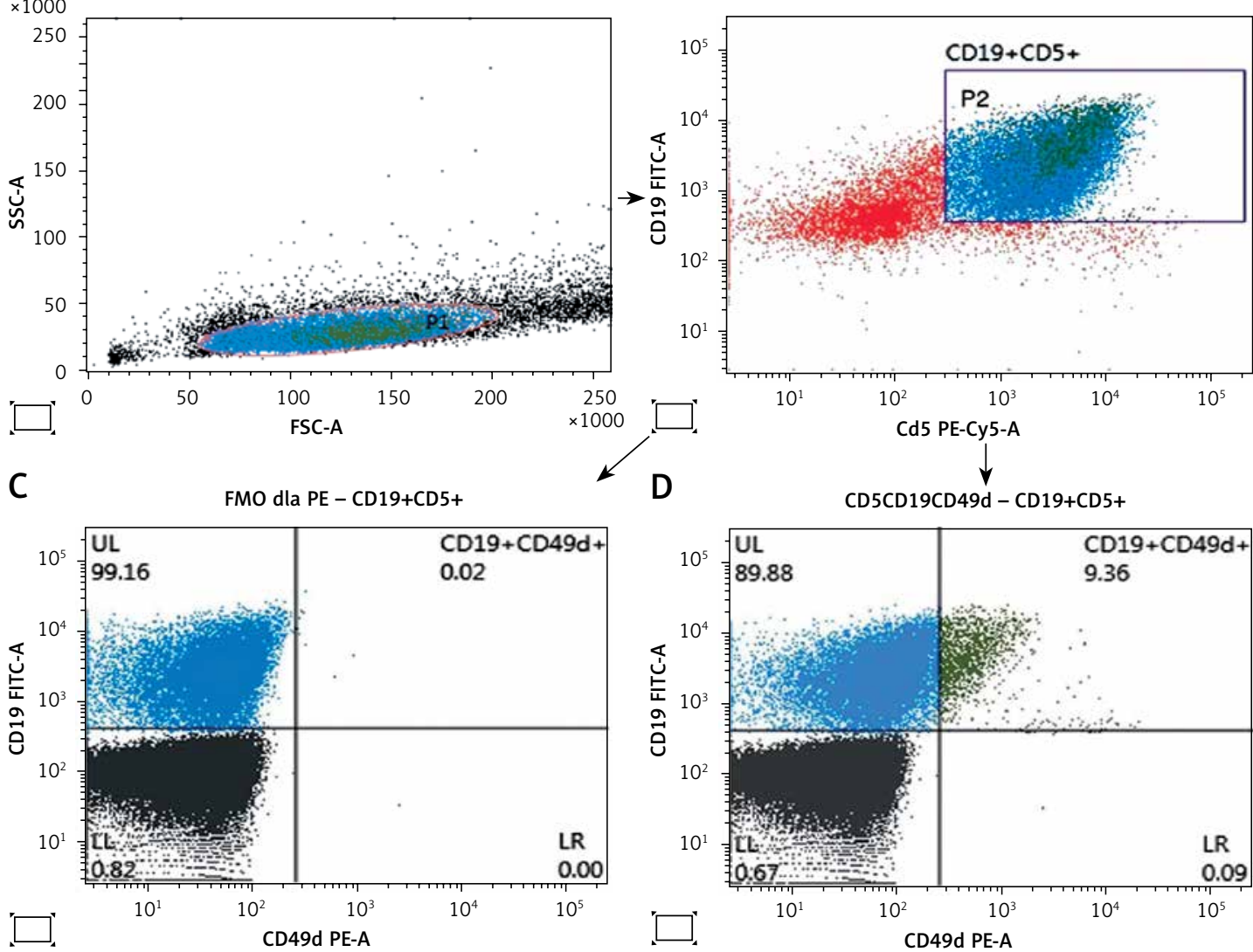

Figure 1. Cytometric analysis of CD49d expression on CD19+CD5+ cells. CD49d expression was normalised against fluorescence minus one (FMO) control. The dot plots (A, B, C, D) represent an example of the analysis of CD49d expression on chronic lymphocytic leukaemia (CLL) cells. A - The lymphocyte population was gated (region P1) based on side vs. forward scatter (FSC vs. SSC) distribution. B - From the P1 region CLL cells were gated (region P2) as double positive cells for CD19 and CD5. CLL were gated (region P2) as double positive cells for CD19 and CD5. C - Compared to FMO control, the CD49d expression on CLL cells was evaluated. D - The final dot plot was acquired using combined gating of events using P1 and P2 regions; thereby, all CD19+CD49d+ cells were CD5+. The final dot plot was acquired using combined gating of events using P1 and P2 regions; thereby, all CD19+CD49d+ cells were $C D 5+$ number in the upper right quadrant in the dot plot represents the percentage of CD19+CD49d+ $(9.36 \%)$ 
ferences between the groups were verified using the Mann-Whitney and Kruskal-Wallis tests, while correlations between the variables were assessed using the Spearman rank correlation coefficient. The Kaplan-Meier method was used to analyse the TTFT of CLL patients. Univariate and multivariate analyses were performed using the Cox regression model. All the analyses assumed a significance level of 0.05 .

\section{Results}

The prognostic value of CD49d expression in chronic lymphocytic leukaemia

To determine the prognostic value of CD49d in CLL, we divided the patients into groups according to the presence of certain prognostic factors. CD49d expression tended to be higher in patients characterised by unmutated (UM) IGHV gene status than in those with mutated (MUT) IGHV (14.33 vs. 5.09, $p=0.151$ ) (Figure 4 A). ZAP-70+ (cut-off of 20\%) patients had higher Cd49d expression than did ZAP-70-patients (Figure 2 A); likewise, CD38+ (cut-off of 30\%) patients tended to have higher expression than did CD38-patients (Figure 2 B). CD49d expression weakly correlated with serum level of $\beta 2$-microglobulin $(r=0.273, p=0.012)$ (Figure $3 \mathrm{~A}$ ) and activity of lactate dehydrogenase (LDH) $(r=0.159, p<0.01)$ (Figure $3 \mathrm{~B})$.

We ascertained whether CD49d expression depended on new molecular markers in CLL: SF3B1, NOTCH1, and MYD88. The analysis revealed a tendency for CD49d to be more highly expressed in MUT SF3B1 cases than in UNMUT SF3B 1 (93.98 vs. 7.86, $p=0.06$ ) (Figure $4 \mathrm{~B}$ ). We observed a tendency in MUT NOTCH1 versus UNMUT NOTCH1 cases (18.52 vs. 7.86, $p=0.140$ ) (Figure 4 C). CD49d expression, however, did not depend on the mutational status of MYD88 (4.65 vs. $11.03, p=0.227$ ) (Figure 4 D). Likewise, CD49d had similar expression in patients for whom the stereotyped B-cell receptor (BCR) sequences provided adverse prognosis (subsets \#1, \#2, and \#8) and those with favourable prognosis (the remaining subsets) (6.42 vs. 8.32, $p=0.484)$.

\section{B}

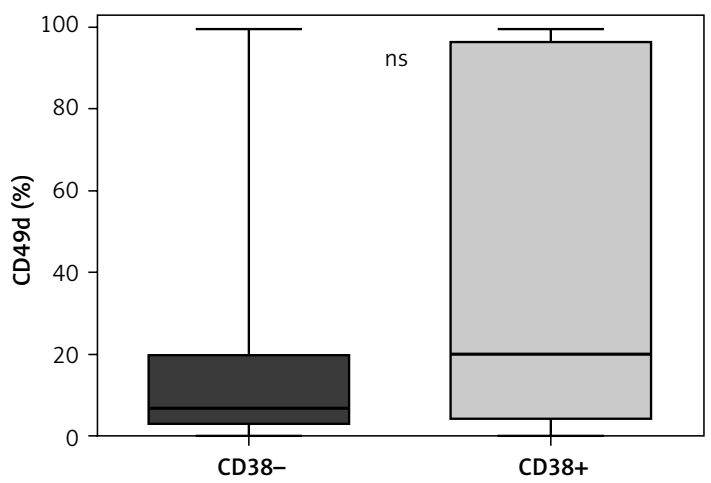

CD38-

CD38+
A

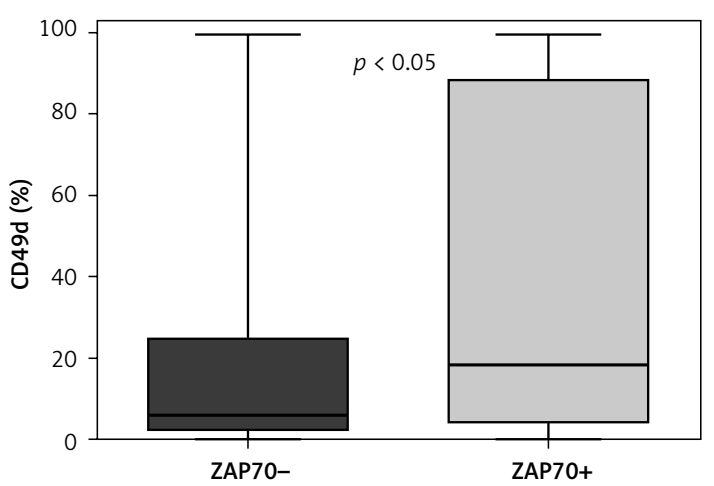

Figure 2. Expression of CD49d protein in patients depending on ZAP-70 (A) and CD38 (B) expression. A - CD49d protein expression was higher in ZAP-70+ patients than in ZAP-70 patients (18.52 vs. $6.57, p=0.028)$. B - CD49d protein expression tends to be higher in CD38+ patients than in CD38 patients (20.48 vs. $7.25, p=0.072$ )

A

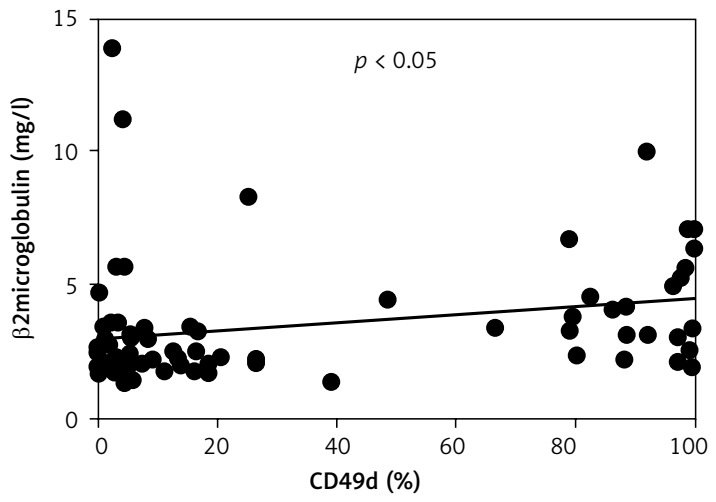

B

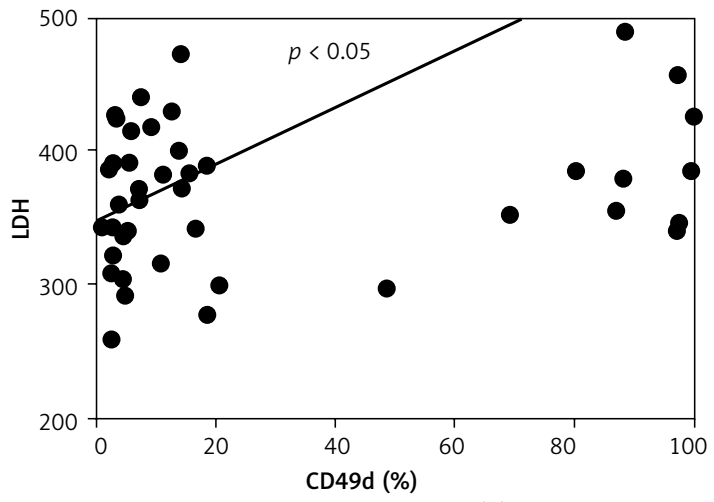

Figure 3. Correlation between CD49d protein expression and the serum level of $\beta 2$-microglobulin (A) and activity of lactase dehydrogenase (LDH) (B). A - Correlation between CD49d protein expression and the serum level of $\beta 2$-microglobulin was low $(r=0.273, p=0.012)$. B - Correlation between CD49d protein expression and the activity of $\mathrm{LDH}$ was also low $(r=0.159, p<0.01)$ 
A

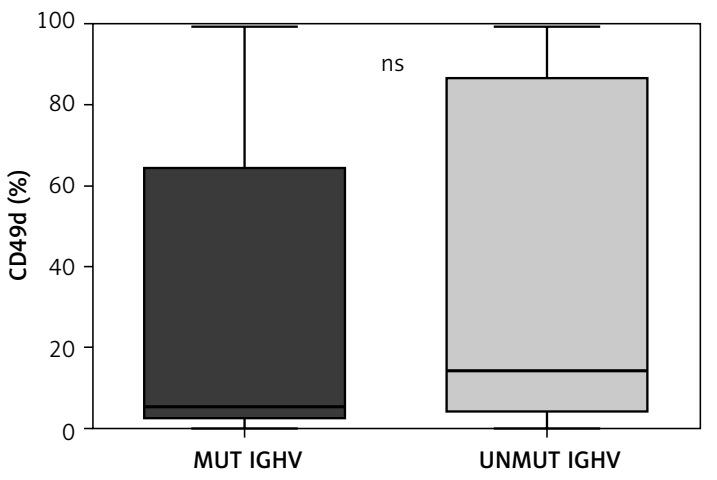

C

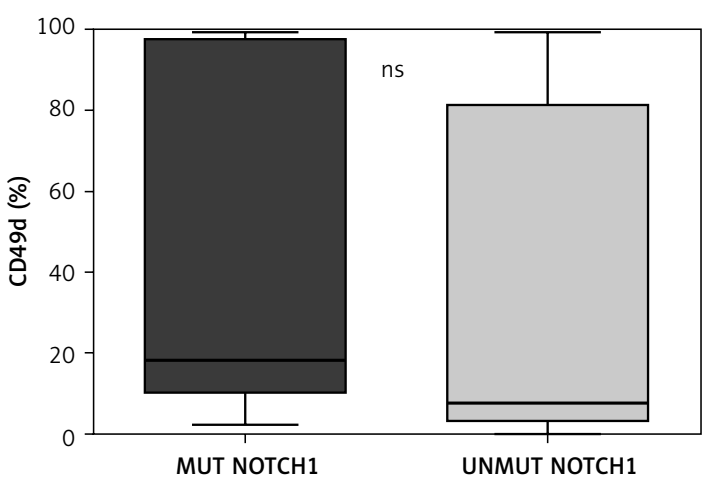

B

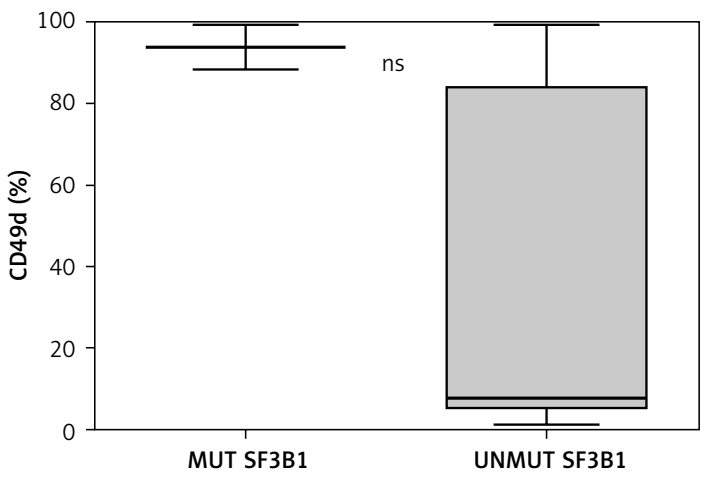

D

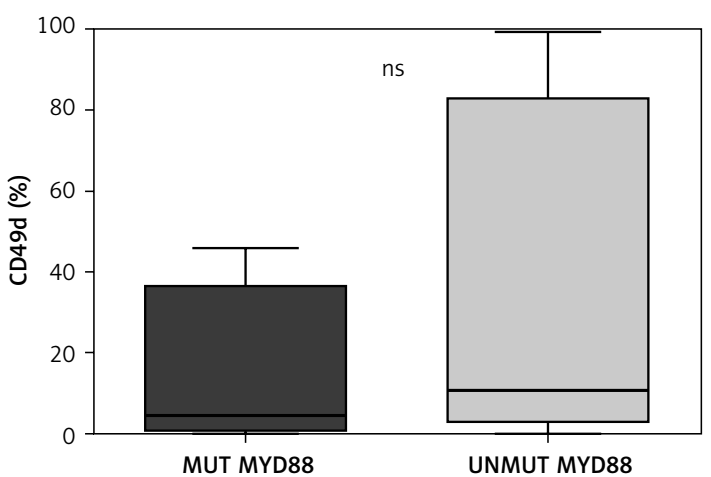

Figure 4. CD49d protein expression depending on mutational status of IGHV (A), SF3B1 (B), NOTCH1 (C), and MYD88 (D). A - Expression of CD49d protein tends to be higher in patients with the UNMUT IGHV gene status than in those with the MUT IGHV gene status (14.33 vs. 5.09, $p=0.151)$. B - Expression of CD49d protein tends to be higher in the MUT SF3B1 patients than in the UNMUT SF3B1 ones (93.98 vs. 7.86, $p=0.06$ ). C - Expression of CD49d protein tends to be higher in the MUT NOTCH1 patients than in the UNMUT NOTCH1 patients (18.52 vs. $7.86, p=0.140$ ). $\mathbf{D}$ - Expression of CD49d protein was similar in MUT MYD88 and UNMUT MYD88 patients (4.65 vs. $11.03, p=0.227)$

The influence of the cut-off on the impact of Cd49d expression on the clinical outcome in chronic lymphocytic leukaemia

As the survival curves show (Figure 5 A), the expressions of ZAP-70 and CD38 proved to be efficient prognostic values in CLL $(p=0.001)$. We divided the patients into 4 groups according to positive and negative expressions of both markers, for which we compared TTFT predictions. We found worse prognosis for patients with ZAP-70+ and CD38+. The recommended cut-off level of CD49d expression is $30 \%$. Because the preliminary analysis of our data suggested other cut-off levels of CD49d expression, we used 3 of them: $30 \%, 20 \%$, and the median (9.9\%). Time to first treatment did not significantly differ in patients with CD49d+ expression and those with CD49d-expression, irrespective of the cut-off point used: $30 \%(p=0.501)$ (Fig. 4B), 20\% ( $p=0.256)$ (Figure $5 \mathrm{C}$ ), and the median of $9.9 \%(p=0.821)$ (Figure $5 \mathrm{D})$.

All variables were assessed in a univariate Cox regression model, and those which were statistically significant were considered for inclusion in multivariate analysis (Table II). There was no sta- tistically significant difference in CD49d protein and mRNA expression in univariate analyses, respectively $(\mathrm{p}=0.51$, hazard ratio $\mathrm{HR}=1.26,95 \%$ $\mathrm{Cl}: 0.6-2.5)$ and $(\mathrm{p}=0.83, \mathrm{HR}=1.16,95 \% \mathrm{Cl}$ : $0.3-4.8)$.

\section{The prognostic value of transcript of CD49d (ITGA4) expression on mRNA levels in chronic lymphocytic leukaemia and its impact on clinical outcome in chronic lymphocytic leukaemia}

To characterise the prognostic value of ITGA4 in CLL, we studied whether ITGA4 expression affected the clinical outcome, defined as TTFT. Time to first treatment did not depend on CD49d expression at the mRNA level ( $p=0.763$ ) (Figure 6). ITGA4 expression also did not show significant prognostic value in the groups of patients divided according to CD38 and ZAP-70 expression factors (Figure $7 \mathrm{~A}, \mathrm{~B}$ ).

\section{Discussion}

Recent advances in CLL research - such as the discovery of its genomic landscape and the 
A

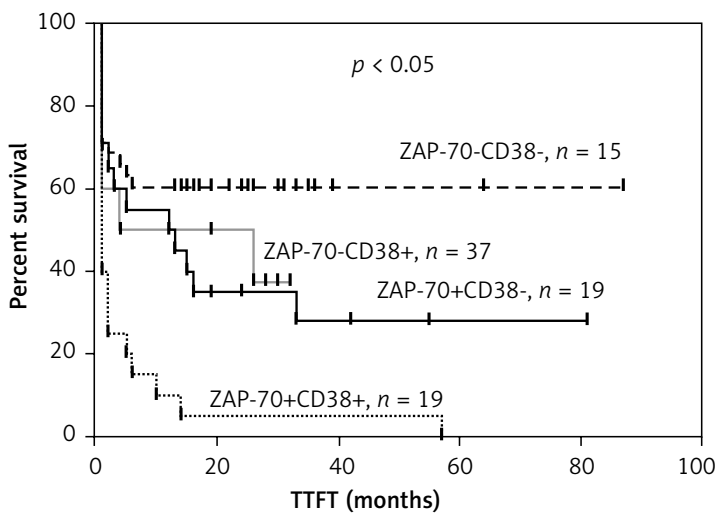

c

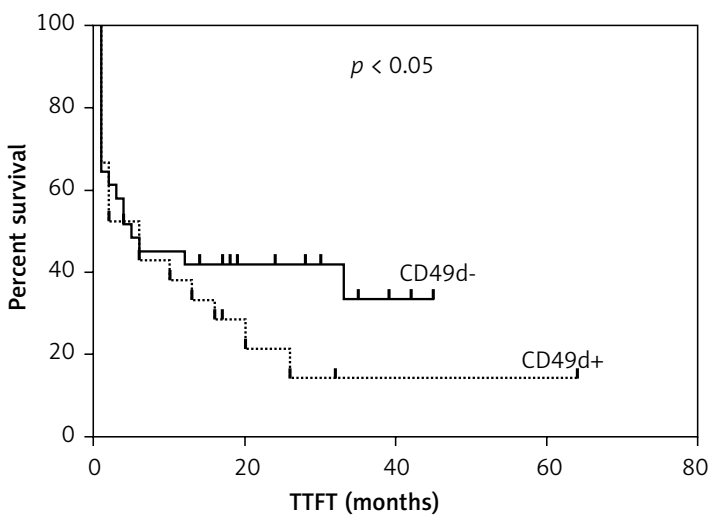

B

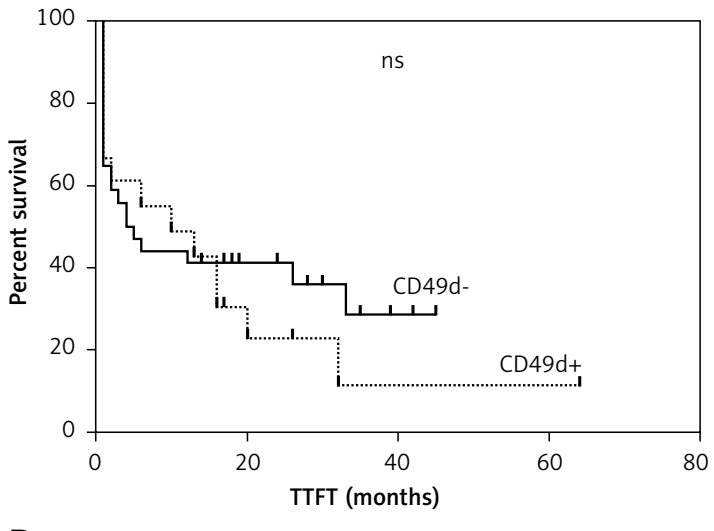

D

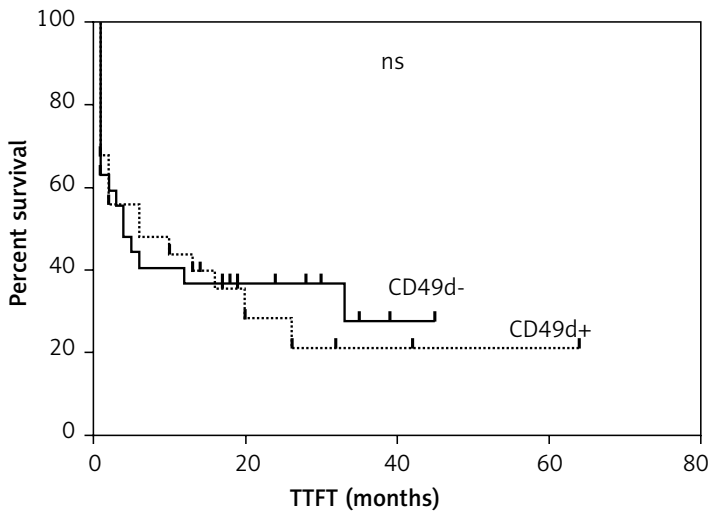

Figure 5. Prognostic values in terms of time to first treatment (TTFT) of ZAP-70 and CD38 expression (A), CD49d protein expression (cut-off 30\%) (B), cut-off of $20 \%$ (C), and the median cut-off of 9.9\% (D). A - There were statistically significant differences in terms of TTFT in patients divided into 4 groups according to positive and negative expressions of ZAP-70 and CD38 ( $p=0.001)$. B - There were no statistically significant differences in terms of TTFT in patients with the positive and negative CD49d protein expression (cut-off $30 \%)(p=0.505)$. $\mathbf{C}-$ There were no statistically significant differences in terms of TTFT in patients with positive and negative CD49d protein expression (cut-off 20\%) ( $p=0.26)$. D - There were no statistically significant differences in terms of TTFT in patients with positive and negative CD49d protein expression (median cut-off of 9.9\%) ( $p=0.821$ )

Table II. Univariate and multivariate Cox regression analysis model

\begin{tabular}{|c|c|c|c|c|c|c|}
\hline \multirow[t]{2}{*}{ Variable } & \multicolumn{3}{|c|}{ Univariate analysis } & \multicolumn{3}{|c|}{ Multivariate analysis } \\
\hline & Hazard ratio & $\mathrm{Cl}(95 \%)$ & $p$-value & Hazard ratio & $\mathrm{Cl}(95 \%)$ & $p$-value \\
\hline Gender & 0.79 & $0.5-1.3$ & 0.36 & & & \\
\hline Age & 1.00 & $1.0-1.0$ & 0.77 & & & \\
\hline SF3B1 gene & 0.40 & $0.1-1.8$ & 0.22 & & & \\
\hline CD49d status & 1.26 & $0.6-2.5$ & 0.51 & & & \\
\hline CD49d mRNA & 1.16 & $0.3-4.8$ & 0.83 & & & \\
\hline $\mathrm{LDH}$ & 1.00 & $1.0-1.0$ & 0.06 & & & \\
\hline$\beta 2$-microglobulin & 1.25 & $1.1-1.4$ & 0.0001 & 1.11 & $0.8-1.5$ & 0.53 \\
\hline ZAP-70 & 2.35 & $1.4-4.1$ & 0.002 & 2.12 & $0.9-5.2$ & 0.10 \\
\hline CD38 status & 2.12 & $1.2-3.6$ & 0.005 & 0.73 & $0.3-1.9$ & 0.52 \\
\hline IGHV status & 4.37 & $2.3-8.3$ & 0.000006 & 2.13 & $0.7-6.5$ & 0.19 \\
\hline NOTCH1 gene & 1.79 & $1.0-3.2$ & 0.05 & 3.44 & $1.3-9.1$ & 0.01 \\
\hline Disease stage at diagnosis & 4.46 & $2.0-10.2$ & 0.0004 & 2.93 & $0.8-10.5$ & 0.10 \\
\hline
\end{tabular}

Cl-confidence interval, SF3B1 - splicing factor 3B subunit 1, LDH-lactate dehydrogenase, ZAP-70-zeta-chain-associated protein kinase $70,1 G H V$ - immunoglobulin heavy-chain variable region gene, NOTCH1 - neurogenic locus notch homologue protein 1 . The cut-off levels used in the analysis were: SF3B1 gene: mutated vs. non mutated; CD49d status: $30 \%$ positive cells; ZAP-70: 20\% positive cells, CD38 status: $30 \%$ positive cells; IGHV status: mutated vs. non mutated, NOTCH1 gene: mutated vs. non mutated; disease stage at diagnosis: RAl scale $0-2$ vs. 3-4. 
A

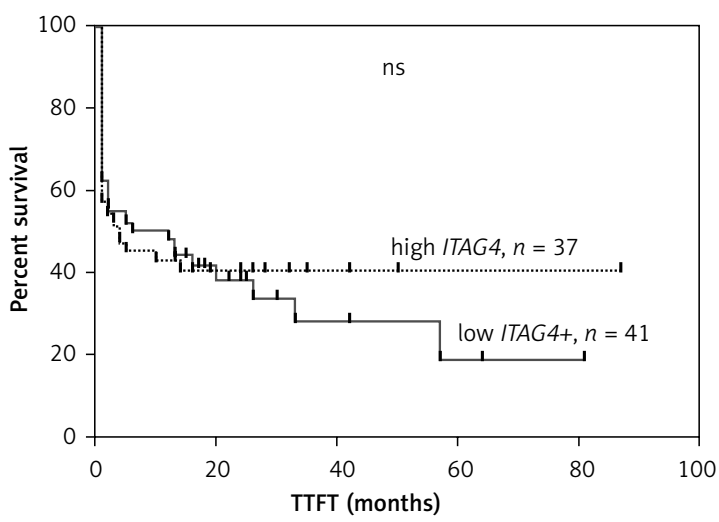

Figure 6. Time to first treatment (TTFT) in patients with low and high ITGA4 expression. There were no statistically significant differences in terms of TTFT in patients with the positive and negative CD49d expression at mRNA level $(p=0.763)$

development of prognostic tests - have changed the way CLL is diagnosed, managed, and treated. The International Workshop on CLL published in 2018 updated recommendations on the diagnosis and management of CLL. Among the changes was an update of the list of potential biomolecular markers with a new potential immunophenotypic marker with clinical relevance: CD49d [3].

In order to learn whether CD49d should be used not only as a potential prognostic marker but also as an effective one, we analysed in detail CD49d expression at both protein and mRNA levels in the context of currently established prognostic factors and TTFT in a representative cohort of CLL patients. Although its prognostic value has already been confirmed at the protein level, this knowledge has yet not been transferred to routine practice [7-9, 12].

According to 2 landmark papers presented in 1999 , one of the most powerful prognostic markers discovered to date is IGHV mutational status $[23,24]$. Chronic lymphocytic leukaemia patients

A

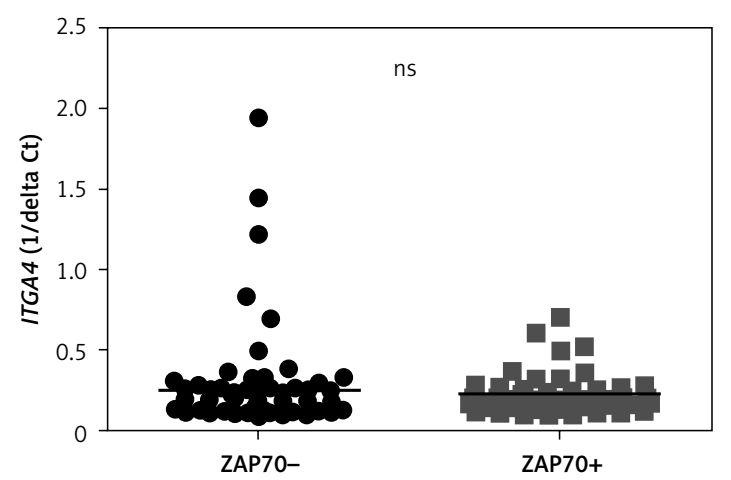

with the MUT IGHV gene usually have a low-risk disease and good prognosis, while those with the UNMUT IGHV genes (defined as $98 \%$ or more sequence homology to the nearest germline gene) usually have progressive disease and respond poorly to therapy, both shortening their survival $[7,23]$. Our study has shown higher CD49d expression in patients with unmutated IGHV status, a result consistent with previous studies that revealed a correlation between CD49d expression and IGHV status [7, 13, 19]. We have also confirmed that CD49d expression correlates with other aggressive biological markers, such as high $\beta 2$-microglobulin and LDH [19]. CD49d has been proven to correlate with the expressions of ZAP70 and CD38, both related with bad prognosis yet another result that is consistent with previous researches [7, 8, 19, 25].

Next-generation sequencing has identified new genomic abnormalities in CLL patients, such as NOTCH1 and SF3B1 mutations. Both have negative prognostic significance, but the SF3B1 mutation has already been accepted as an independent prognostic factor [22]. Affecting up to $15 \%$ CLL patients and significantly overrepresented in the trisomy 12 cytogenetic, NOTCH1 mutations are known to show poor prognosis [26]. Moreover, Benedetti et al. showed that CD49d expression correlates with NOTCH1 mutation, indicating that CD49d expression can be up-regulated by the activation of the NOTCH1 pathway through the involvement of NF-kB [20]. In our study, CD49d expression tended to be higher in patients with NOTCH1 mutations and those with SF3B1 mutations. Our data partly corroborate the results of Baumann et al. [7], who showed a significant correlation between CD49d and both mutations, while we have observed a tendency. In our study, we did not show an association between CD49d expression and MYD88 mutations, a result contributing to various data indicating that this ab-

B

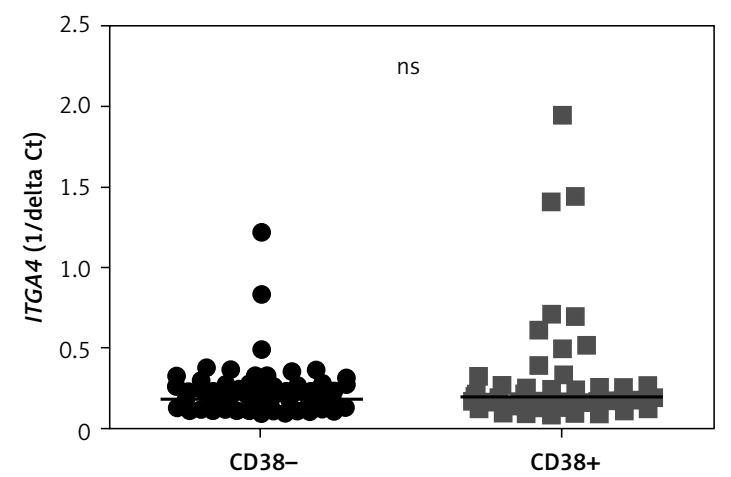

Figure 7. ITGA4 expression in patients with negative and positive expression of ZAP-70 (A) and CD38 (B). A - Expression of CD49d at mRNA level was similar in ZAP-70-positive and ZAP-70-negative patients (0.1859 vs. 0.1894, $p=0.988)$. B - Expression of CD49d at mRNA level was similar in CD38-positive and CD38-negative patients (0.1976 vs. $0.1869, p=0.295)$ 
erration has no clinical implication in CLL patients [27-29]. Some studies have reported longer TTFT and overall survival in CLL patients with MYD88 mutations [30]; however, the relevance of MYD88 mutation remains unclear and requires further investigation.

We used TTFT to analyse the prognostic value in the context of the patients' clinical outcomes. TTFT not show significant differences for any of the assessed cut-off levels: 30\%, 20\%, and the median (9.9\%). On the other hand, analysing TTFT against the background of currently accepted immunophenotypic factors revealed significant differences in the CD38 and ZAP-70 subgroups, with worse prognosis in positive patients in both subgroups (ZAP$70+$ and CD38+). The univariate and multivariate Cox regression model also did not prove the negative prognostic value of CD49d, despite results of analyses of other prognostic factors which confirmed that our cohort included a representative group of CLL patients. These data disagree with previous reports that pointed out negative clinical implications (TTFT and OS) of high CD49d expression in CLL patients [7, 8, 19]. Moreover, in the study by Bulian et al. CD49d emerged as the strongest flow cytometry-based prognostic marker, even better than CD38 and ZAP-70 [8].

CD49d may also be expressed in a bimodal pattern in CLL patients with the copresence of negative and positive groups of cells in the same sample. Recent multicentre research showed that CLL patients with CD49d expression exceeding the $30 \%$ cut-off as well as with bimodal expression of CD49d had negative clinical implications. Both subpopulations, regardless of the $30 \%$ cut-off in a bimodal pattern of expression, experienced similar clinical behaviour in the context of a chemoimmunotherapy and ibrutinib setting [31].

Targeting CD49d could represent an interesting approach in the management of CLL. The monoclonal humanised IgG4 anti-CD49d antibody - natalizumab - demonstrated the potential to overcome stromal cell-induced resistance of B cell lymphoma cells against rituximab and cytotoxic drugs in vitro [9, 32]. CD49d playing a key role in adhesion and migration of cells and is related with BCR signalling by inside-out activation [10]. To date, the BCR signalling pathway has emerged as one of the most important targets for novel therapies in CLL using BCR kinase inhibitors, such as ibrutinib [33]. The therapeutic effects of BCR inhibitors in CLL may contribute to similar clinical activity of integrin inhibitors. It is also hypothesised that these agents could synergise with chemotherapeutic agents and monoclonal antibodies [9, 33].

Flow cytometry is the common laboratory technique; however, it is associated with disadvantages such as inconsistent performance depending on the monoclonal antibodies and fluorochromes used and different cut-off levels reported. The expression of CD49d could also be assessed by qRT-PCR, which is a widely used, sensitive, and accurate method for monitoring gene expression [34]. Another prognostic marker in CLL patients - the expression of ZAP-70 was confirmed to have useful prognostic value both in protein and mRNA levels [35]. Therefore, we performed an analysis of CD49d expression using both methods. In our study, we results of expression of CD49d analysed by Quantitative reverse transcriptase PCR (CD49d mRNA) was compared to other prognostic factors and have showed no statistical difference.

On the other hand analysis of CD49d expression by cytometry method showed statistical difference comparing to other prognostic factors in CLL. That's why cytometry method should be used to assess prognostics of CLL results may contribute to numerous post-transcriptional and translational regulation mechanisms in protein expression, such as phosphorylation, ubiquitination, methylation, acetylation, or GlcNAcylation [36].

In conclusion, this study has shown that high CD49d expression is associated with other aggressive biological markers in CLL patients, confirming what previous studies have suggested, i.e. that CD49d is a promising novel prognostic marker.

\section{Acknowledgements}

This research was supported by the Polish Scientific Centre funds of grant NCN 2018/29/B/ NZ5/02706, Young Section of the Polish Society of Hematologists and Transfusiologists, Medical University of Lublin grants: No. MNmb 536, DS462, and Own Scholarship Fund.

\section{Conflict of interest}

The authors declare no conflict of interest.

\section{References}

1. Hallek M. Chronic lymphocytic leukemia: 2017 update on diagnosis, risk stratification, and treatment. Am J Hematol 2017; 92: 946-65.

2. Yosifov DY, Wolf C, Stilgenbauer S, Mertens D. From biology to therapy: the CLL success story. Hemasphere 2019; 3: e175.

3. Hallek M, Cheson BD, Catovs.ky D, et al. iwCLL guidelines for diagnosis, indications for treatment, response assessment, and supportive management of CLL. Blood 2018; 131: 2745-60.

4. Giannopoulos K. Biology and prognosis in chronic lymphocytic leukemia. Acta Haematol Pol 2010; 41: 433-40.

5. Kalinka E, Drozd-Sokołowska J, Waszczuk-Gajda A, et al. Hepatitis B virus screening in patients with non-Hodgkin lymphoma in clinical practice in Poland - a report of the Polish Lymphoma Research Group. Arch Med Sci 2020; 16: 157-61. 
6. Mertens D, Bullinger L, Stilgenbauer S. Chronic lymphocytic leukemia - genomics lead the way. Haematologica 2011; 96: 1402-5

7. Baumann T, Delgado J, Santacruz R, et al. CD49d (ITGA4) expression is a predictor of time to first treatment in patients with chronic lymphocytic leukaemia and mutated IGHV status. Br J Haematol 2016; 172: 48-55.

8. Bulian P, Shanafelt TD, Fegan C, et al. CD49d is the strongest flow cytometry - based predictor of overall survival in chronic lymphocytic leukemia. J Clin Oncol 2014; 32: 897.

9. Dal Bo M, Tissino E, Benedetti D, et al. Functional and clinical significance of the integrin alpha chain CD49d expression in chronic lymphocytic leukemia. Curr Cancer Drug Targets 2016; 16: 659-68.

10. Kriston C, Plander M, Márk Á, et al. In contrast to high CD49d, low CXCR4 expression indicates the dependency of chronic lymphocytic leukemia (CLL) cells on the microenvironment. Ann Hematol 2018; 97: 2145-52.

11. Mahmoud MS, Salaheldin EM, Sayed NG, Solyman AF. Association of CD49d and CD44 in chronic lymphocytic leukemia patients and their effects on response to fludarabine, cyclophosphamide, and rituximab therapy. J Curr Med Res Pract 2018; 3: 147-53.

12. Rose DM, Han J, Ginsberg MH. 4 integrins and the immune response. Immunol Rev 2002; 186: 118-24.

13. Rossi D, Zucchetto A, Rossi FM, et al. CD49d expression is an independent risk factor of progressive disease in early stage chronic lymphocytic leukemia. Haematologica 2008; 93: 1575-9.

14. Shanafelt TD, Geyer SM, Bone ND, et al. CD49d expression is an independent predictor of overall survival in patients with chronic lymphocytic leukaemia: a prognostic parameter with therapeutic potential. $\mathrm{Br} J$ Haematol 2008; 140: 537-46.

15. Pasikowska M, Walsby E, Apollonio B, et al. Phenotype and immune function of lymph node and peripheral blood CLL cells are linked to transendothelial migration. Blood 2016; 128: 563-73.

16. Till KJ, Spiller DG, Harris RJ, Chen H, Zuzel M, Cawley JC. $\mathrm{CLL}$, but not normal, $\mathrm{B}$ cells are dependent on autocrine VEGF and alpha4beta 1 integrin for chemokine-induced motility on and through endothelium. Blood 2005; 105: 4813-19.

17. Strati P, Parikh SA, Chaffee KG, et al. CD49d associates with nodal presentation and subsequent development of lymphadenopathy in patients with chronic lymphocytic leukaemia. Br J Haematol 2017; 178: 99-105.

18. Duchartre Y, Bachl S, Kim HN, et al. Effects of CD49d-targeted antisense-oligonucleotide on $\alpha 4$ integrin expression and function of acute lymphoblastic leukemia cells: results of in vitro and in vivo studies. PLOS One 2017; 12: e0187684.

19. Zucchetto A, Vaisitti T, Benedetti D, et al. The CD49d/ CD29 complex is physically and functionally associated with CD38 in B-cell chronic lymphocytic leukemia cells. Leukemia 2012; 26: 1301-12.

20. Benedetti D, Tissino E, Pozzo F, et al. NOTCH1 mutations are associated with high CD49d expression in chronic lymphocytic leukemia: link between the NOTCH1 and the NF- $\mathrm{B}$ B pathways. Leukemia 2018; 32: 654-62.

21. Hus I, Podhorecka M, Bojarska-Junak A, et al. The clinical significance of ZAP-70 and CD38 expression in B-cell chronic leukemia. Ann Oncol 2006; 17: 683-90.

22. Putowski $M$, Podgórniak $M$, Piróg $M$, et al. Prognostic impact of NOTCH1, MYD88, and SF3B1 mutations in Polish patients with chronic lymphocytic leukemia. Pol Arch Intern Med 2017; 127: 238-44.
23. Damle RN, Wasil T, Fais F, et al. Ig V gene mutation status and CD38 expression as novel prognostic indicators in chronic lymphocytic leukemia. Blood 1999; 94: 1840-7.

24. Hamblin TJ, Davis Z, Gardiner A, Oscier DG, Stevenson FK. Unmutated Ig $\mathrm{V}(\mathrm{H})$ genes are associated with a more aggressive form of chronic lymphocytic leukemia. Blood 1999; 94: 1848-54.

25. Gattei V, Bulian P, Del Principe MI, et al. Relevance of CD49d protein expression as overall survival and progressive disease prognosticator in chronic lymphocytic leukemia. Blood 2008; 111: 865-73.

26. Balatti V, Bottoni A, Palamarchuk A, et al. NOTCH1 mutations in CLL associated with trisomy 12. Blood 2012; 119: 329-31.

27. Baliakas P, Hadzidimitriou A, Sutton LA, et al. European Research Initiative on CLL (ERIC): recurrent mutations refine prognosis in chronic lymphocytic leukemia. Leukemia 2015; 29: 329-36.

28. Jeromin S, Weissmann S, Haferlach C, et al. SF3B1 mutations correlated to cytogenetics and mutations in NOTCH1, FBXW7, MYD88, XPO1 and TP53 in 1160 untreated CLL patients. Leukemia 2014; 28: 108-17.

29. Rossi D, Rasi S, Spina V, et al. Integrated mutational and cytogenetic analysis identifies new prognostic subgroups in chronic lymphocytic leukemia. Blood 2013; 121: 1403-12.

30. Martínez-Trillos A, Pinyol M, Navarro A, et al. Mutations in TLR/MYD88 pathway identify a subset of young chronic lymphocytic leukemia patients with favorable outcome. Blood 2014; 123: 3790-6.

31. Tissino E, Pozzo F, Benedetti D, et al. CD49d promotes disease progression in chronic lymphocytic leukemia: new insights from CD49d bimodal expression. Blood 2020; 135: 1244-54.

32. Mraz M, Zent CS, Church AK, et al. Bone marrow stromal cells protect lymphoma B-cells from rituximab-induced apoptosis and targeting integrin alpha-4-beta-1 (VLA4) with natalizumab can overcome this resistance. $\mathrm{Br} J$ Haematol 2011; 155: 53-64.

33. Herman SE, Mustafa RZ, Jones J, et al. Treatment with ibrutinib inhibits BTK- and VLA-4-dependent adhesion of chronic lymphocytic leukemia cells in vivo. Clin Cancer Res 2015; 21: 4642-51.

34. Adams RLC, Cheung C, Banh R, et al. Prognostic value of ZAP-70 expression in chronic lymphocytic leukemia as assessed by quantitative polymerase chain reaction and flow cytometry. Cytometry B Clin Cytom 2014; 86: 80-90.

35. Wang YH, Zou ZJ, Liu L, et al. Quantification of ZAP-70 mRNA by real-time PCR is a prognostic factor in chronic lymphocytic leukemia. J Cancer Res Clin Oncol 2012; 138: 1011-7.

36. Greenbaum D, Colangelo C, Williams K, Gerstein M Comparing protein abundance and mRNA expression levels on a genomic scale. Genome Biol 2003; 4: 117. 